\title{
Eclosion et multiplication de Heterodera schachtii Schmidt en présence de colza ou de radis four- ragers
}

\author{
Georges CAUBEL \& Bernard CHAUBET \\ I.N.R.A., Laboratoire de Zoologie, Centre de Recherches de Rennes, B.P. 29, F 35650 Le Rheu
}

RÉSUMÉ

Les progrès de la sélection à l'égard de la résistance à Heterodera schachtii, s'ils n'ont pas encore abouti à la création de variétés commerciales de betteraves sucrières, ont permis récemment la mise sur le marché étranger et français de variétés de crucifères - radis fourragers et moutardes - utilisables comme culture dérobée en engrais vert.

Le comportement des variétés de radis fourrager "Slobolt», "Pegletta " et «Siletina " et du colza "Crésor » a été étudié comparativement. La résistance s'exprime au niveau de l'importance de la multiplication de $H$. schachtii sur les radicelles de plantes cultivées en pots, en conditions semi-contrôlées (inoculum et milieu).

Le colza est plus favorable à la multiplication du nématode que les radis fourragers chez lesquels des différences marquées sont observées entre variétés : avec «Pegletta », le nombre de femelles formées est beaucoup plus faible qu'avec «Siletina » (44 fois) ou le colza (150 fois), tandis que le pourcentage de plantes qui permettent la formation de femelles demeure toujours très faible pour "Pegletta " comparativement à celui des autres radis. Par contre, la stimulation en présence de jeunes plantes de la sortie des larves embryonnées contenues dans les kystes s'observe indistinctement quelle que soit la variété utilisée mais montre des différences selon les origines des kystes utilisés.

Encore préliminaire, la méthodologie expérimentée permet de déterminer chez des variétés de crucifères leur niveau de résistance à $H$. schachtii.

Mots clés additionnels : Résistance, Raphanus sativus $L$. ssp. oleifera (D.C.) Metzg., Brassica napus L. ssp. oleifera (Metzg.) Sinsk.

Hatching and multiplication of beet cyst nematode Heterodera schachtii Schmidt on oilseed rape and fodder radish

Commercial sugar beet resistant to beet cyst nematode is not available, but some resistant cruciferous green manure crops have now been developed in Germany and in the Netherlands. The resistance known in fodder radish cvs. "Slobolt » and «Pegletta » was compared with susceptible cv. "Siletina » and with oilseed rape «Crésor ». Multiplication of Heterodera schachtii on roots of plants grown in pots in standard conditions of inoculum was lower on fodder radish than on oilseed rape ; there were differences between cultivars of radish. Numbers of females developed on "Pegletta » were lower (44 times) than on "Siletina " or "Crésor " (150 times). The percentage of plants with females was always very low on "Pegletta " compared with the other radishes. Moreover, stimulation of larval hatching in water in contact with plantlets was observed with all these crucifers; the quality of the cysts influenced the level of hatching and the stimulating effect of root diffusates. This kind of test could be useful to determine the level of resistance of cruciferous cultivars.

Additional key words : Resistance, Raphanus sativus L. ssp. oleifera (D.C.) Metzg., Brassica napus L. ssp. oleifera (Metzg.) Sinsk.

\section{INTRODUCTION}

Le nématode à kyste de la betterave, Heterodera schachtii Schmidt, est considéré comme l'espèce la plus nuisible à la production sucrière en France (CAUBEL, 1982). Les travaux de création de variétés de bet- teraves résistantes à ce ravageur, entrepris dans plusieurs pays étrangers, ne semblent pas devoir aboutir à la création d'une variété commerciale avant plusieurs années. Depuis peu, des cultivars de crucifères s'opposant à la multiplication de $H$. schachtii ont été mis en évidence (BAUKLOCH, 1976 ; LUBBERTS \& TOXOPEUS, 
1982) et une sélection active, en cours à l'étranger, permet d'ores et déjà la commercialisation de radis fourragers ou radis oléifères (Raphanus sativus L. ssp. oleifera (D.C.) Metzg.) et de moutardes blanches (Sinapis alba L.) résistants à $H$. schachtii. Leur utilisation en lutte intégrée en Allemagne et en Hollande, a donné des résultats intéressants (MÜLLER \& VON KRIES, 1981 ; HeIJBROEK, 1982 ; STEUdEL \& MÜLLER, 1983).

Pour avoir une action qui appauvrisse la contamination des sols, il est absolument indispensable que ces variétés qui ne permettent pas la multiplication du ravageur visé, stimulent aussi la sortie de ses larves contenues dans les kystes, sinon l'emploi de crucifères comme engrais vert ne se justifie pas aux plans agronomique et phytosanitaire.

C'est pourquoi il nous a paru intéressant d'étudier comparativement les caractéristiques de telles variétés sur 2 aspects biologiques essentiels, "l'éclosion", c'est-à-dire la sortie des larves contenues dans les kystes, et la formation des femelles dans les racines, en recourant à des techniques expérimentales simples et moins contraignantes qu'une inoculation artificielle de cultures de racines en boîte de Petri (Múller, 1978) ou qu'un test de sélection applicable sur de grandes séries de plantes (LUBBERTS \& TOXOPEUS, 1982).

\section{MATÉRIEL ET MÉTHODES}

Les expérimentations d'éclosion et de multiplication sont menées simultanément dans les mêmes conditions de serre, avec le même matériel animal et végétal :

-2 populations d' $H$. schachtii ont été collectées dans des parcelles de betterave à l'automne, l'une, dite souche $\mathrm{Be} 8$, à Concevreux (Aisne), l'autre dite souche Be 10, à Châlons-sur-Marne (Marne). Parmi les kystes extraits du sol en hiver, seuls ceux qui paraissent pleins sont retenus ; ils sont conservés à $7{ }^{\circ} \mathrm{C}$ environ jusqu'à leur utilisation au printemps suivant ;

- les variétés de crucifères utilisées comportent un colza de printemps (Brassica napus L. ssp. oleifera (Metzg.) Sinsk.), "Crésor », et 3 radis fourragers, "Slobolt ", «Pegletta » et "Siletina »; parmi ceuxci, les 2 derniers sont inscrits au catalogue français respectivement depuis 1983 et 1984, tandis que "Slobolt » et " Pegletta » sont signalés comme présentant une résistance à l'égard de $H$. schachtii. Les semences proviennent du laboratoire "Colza » de la Station d'Amélioration des Plantes, I.N.R.A., Le Rheu.

L'essai d'éclosion est réalisé à l'aide de sachets de tulle contenant chacun 10 kystes et maintenus immergés dans $4 \mathrm{ml}$ d'eau du robinet. L'ensemble des flacons est placé en serre à $20^{\circ} \mathrm{C}$ dans une enceinte transparente afin de recevoir la lumière et d'éviter une évaporation trop intense.

Après une semaine pendant laquelle les kystes se réhydratent et les quantités de larves qui en émergent sont pratiquement négligeables et comparables, chaque récipient, à l'exception des témoins, reçoit 3 jeunes plantes d'une variété donnée, obtenues après $5 \mathrm{j}$ de germination sur perlite.
Tous les $7 \mathrm{j}$, durant 10 semaines consécutives, les larves écloses sont recueillies et dénombrées, l'eau et les jeunes plantes renouvelées.

Le test de multiplication est mené selon une technique voisine de celle utilisée par RIVOAL et al. (1978) pour $H$. avenae. Des pots cubiques de $9 \mathrm{~cm}$ de côté, sont remplis chacun environ $400 \mathrm{~g}$ d'un mélange terreux composé de 60 p. 100 de sable de Loire tamisé à $2 \mathrm{~mm}$ et 40 p. 100 de terre franche tamisée à $5 \mathrm{~mm}$. L'inoculum, constitué par un sachet renfermant 50 kystes, est apporté au moment du repiquage des jeunes plantes qui s'effectue à raison de 3 par pot. 20 répétitions sont mises en place avec la souche Be 10 , seulement 10 avec $\mathrm{Be} 8$. Les pots de culture sont disposés dans une serre dont la température oscille autour de $20^{\circ} \mathrm{C}$. Deux mois après l'introduction des kystes dans le substrat, l'extraction des femelles blanches et des kystes nouvellement formés s'effectue en lavant le sol humide et les racines directement dans l'élutriateur de KORT (1960). L'ensemble de l'éluat est réparti dans plusieurs coupelles pour dénombrement sous microscope stéréoscopique.

Le broyage des kystes contenus dans les sachets de l'inoculum permet de dénombrer les œufs et les larves qu'ils renferment encore, aux fins d'évaluer le potentiel infectieux initial total et d'apprécier le pourcentage d'éclosions.

\section{RÉSULTATS}

En ce qui concerne l'éclosion, il apparaît une différence marquée entre les 2 souches de nématodes utilisées puisque le contenu larvaire total par kyste, calculé en additionnant les larves écloses aux œufs et larves dénombrées en fin d'expérience, est de 39 larves pour la souche «Be 8 " et 120 pour « $\mathrm{Be} 10$ » (tabl. 1). De plus, cette dernière population donne, d'une manière générale, un plus fort pourcentage de larves qui éclosent, quel que soit le traitement. Néanmoins, l'allure générale des courbes de sortie des larves est semblable, quelle que soit la souche (fig. 1).

TABLEAU

Eclosion dans l'eau de kystes appartenant à 2 souches d'H. schachtii, en présence de plantules de crucifères. Contenu larvaire des kystes et proportions de larves écloses après 11 semaines.

Hatching of cysts from $2 \mathrm{H}$. schachtii populations in water with cruciferous plants. Larval content and percentage of hatch after 11 weeks.

\begin{tabular}{llcc}
\hline $\begin{array}{c}\text { Souche } \\
\text { H. schachtii }\end{array}$ & Traitement & $\begin{array}{c}\text { Contenu larvaire } \\
\text { moyen d'un kyste }\end{array}$ & $\begin{array}{c}\text { Juvéniles écloses } \\
(\%)\end{array}$ \\
\hline & eau & 40 & 24,1 \\
& Crésor & 40 & 42,8 \\
«Be $8 \%$ & Siletina & 32 & 63,4 \\
& Pegletta & 43 & 57,4 \\
& Moyenne & 39 & 46,3 \\
\hline & eau & 136 & 87,6 \\
& Crésor & 130 & 82,1 \\
& Siletina & 112 & 99,6 \\
& Pegletta & 100 & 97,5 \\
& Moyenne & 120 & 90,9 \\
\hline
\end{tabular}


éclosion

hatching
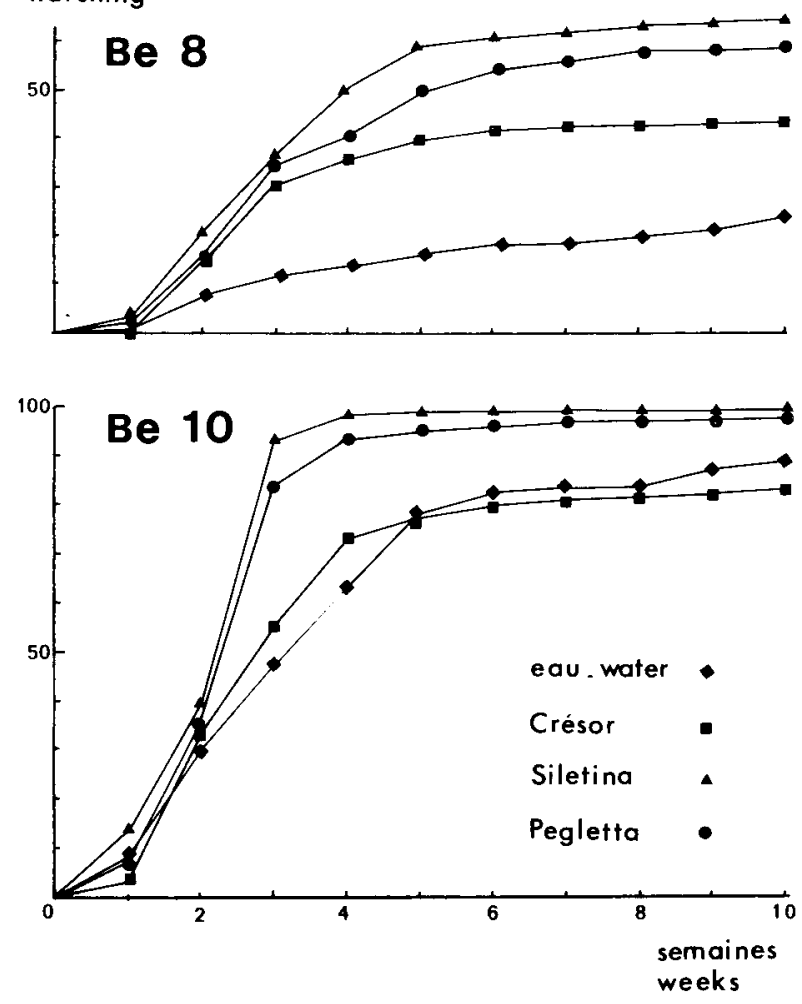

Figure 1

Eclosion dans l'eau de kystes appartenant à 2 souches d'H. schachtii en présence de jeunes plantes de crucifères. Effectifs cumulés exprimés en p. 100 du contenu larvaire total.

Cumulative hatching in water of 2 populations of $\mathrm{H}$. schachtii with plantlets of some cultivars. Percentages of larvae from total viable content.

C'est après 4 à 5 semaines que la quantité de larves écloses atteint 50 p. 100 des effectifs obtenus au bout de 10 semaines. On enregistre parfois entre les répétitions une hétérogénéité qui reste inexpliquée, aussi bien pour le contenu larvaire total que pour le pourcentage d'éclosion.

La présence de crucifères expérimentées stimule la sortie des larves. Cette stimulation est nette, alors que l'éclosion naturelle dans l'eau reste réduite $(\mathrm{Be} 8)$. Aucune différence marquée n'est décelée entre les variétés testées. Les variétés résistantes «Slobolt» et «Pegletta» se comportent à cet égard comme les variétés hôtes.

La multiplication, évaluée par le nombre de femelles formées, diffère selon les 2 souches utilisées. Avec la souche «Be 8 » de nombreuses plantes ne portent pas de femelles, alors qu'avec la souche «Be 10 » le test est beaucoup plus discriminant avec un nombre moyen de femelles toujours supérieur. Ce résultat concorde avec les observations réalisées concernant l'éclosion. Si la comparaison des moyennes (tabl. 2) et des données extrêmes permet d'établir un classement, des différences tranchées apparaissent nettement en ce qui concerne le nombre de pots où un nombre très faible ou nul de femelles est trouvé (tabl. 3). Ainsi, le colza est un hôte plus adapté que les radis fourragers testés. Le radis " Pegletta " n'est pas favorable à la multiplication de $H$. schachtii, puisque plus de la moi-
TABLEAU 2

Multiplication de 2 souches d'H. schachtii sur quelques variétés de crucifères. Effectifs moyens et extrêmes observés par pot.

Multiplication of 2 populations of $\mathrm{H}$. schachtii on some cultivars of Cruciferae. Means populations observed and extreme values.

\begin{tabular}{llclcl}
\hline \hline \multirow{2}{*}{$\begin{array}{c}\text { Souche } H \text {. schachtii } \\
\text { multiplication }\end{array}$} & \multicolumn{2}{c}{ Be 8} & \multicolumn{2}{c}{ Be 10 } \\
& Moyenne & Bornes & Moyenne & Bornes \\
\hline Colza & Crésor & $142,1 \mathrm{a}$ & $3-518$ & $207,2 \mathrm{~s}$ & $4-787$ \\
Radis fourrager & Siletina & $24,1 \mathrm{~b}$ & $1-90$ & $56,8 \mathrm{t}$ & $5-202$ \\
& Slobolt & $16,8 \mathrm{c}$ & $1-112$ & $19,7 \mathrm{tu}$ & $1-99$ \\
& Pegletta & $1,8 \mathrm{~d}$ & $1-4$ & $1,3 \mathrm{v}$ & $1-1 \mathrm{l}$ \\
\hline
\end{tabular}

Les nombres suivis d'une même lettre ne sont pas statistiquement différents, selon le test de rang de Kruskal \& Wallis $(P=0,05)$. Values not followed by the same letter differ significantly according to KRUSKAL \& WaLLIS range test $(\mathbf{P}=0,05)$.

\section{TABLEAU 3}

Multiplication d'H. schachtii (souche Be 10) sur quelques variétés de crucifères. Fréquences, en p. 100 pour chaque variété, du nombre de cas où l'on dénombre 0, 1 ou 2 femelles par pot.

Multiplication of $\mathrm{H}$. schachtii ( $«$ Be $10 »$ population) on different cultivars \% occurrence of zero, one or two females per pot.

\begin{tabular}{llccc}
\hline \hline \multirow{2}{*}{ Cultivars } & & \begin{tabular}{c} 
Nombre \\
moyen \\
\cline { 3 - 5 }
\end{tabular} & $\begin{array}{c}\text { de } \\
\text { femmes }\end{array}$ & Pourcentages de pots avec : \\
\cline { 4 - 5 } Colza & Crésor & 207 & 0 & 0 \\
Radis fourrager & Siletina & 57 & 0 & 5 \\
& Slobolt & 20 & 0 & 5 \\
& Pegletta & 1,3 & 55 & 30 \\
\hline
\end{tabular}

tié des plantes ne portent aucun nématode femelle et si quelques plantes permettent une multiplication, celle-ci reste toujours faible.

\section{DISCUSSION}

La réalisation simultanée des 2 essais a permis de montrer l'importance de l'origine des souches utilisées. Les kystes de ces 2 populations proviennent de 2 parcelles différentes; ils n'ont pas le même contenu larvaire et leurs larves n'éclosent pas avec la même intensité.

Les exsudats racinaires des crucifères testées stimulent l'éclosion d' $H$. schachtii. Cette stimulation est nette lorsque l'éclosion naturelle dans l'eau reste limitée. Ces résultats confirment ceux de STEELE et al. (1982) obtenus avec le colza " Jet Neuf » et des variétés résistantes ou non de radis et de moutarde, mais la comparaison ne peut être complète puisque les noms des variétés utilisées ne sont pas mentionnés par ces auteurs. Après la sortie des larves, la phase de pénétration dans les racines est décisive pour l'accomplissement du cycle biologique.

Dans l'essai en pot, la technique utilisée permet de dénombrer les femelles et les kystes néoformés en les séparant aisément des kystes initiaux. Les conditions semi-contrôlées du test donnent la possibilité d'obtenir 
un taux de multiplication satisfaisant sur la variété de colza «Crésor ». Après le délai de 11 semaines, on observe une grosse majorité de femelles blanches mais de rares kystes et quelques stades larvaires.

\section{CONCLUSION}

Malgré le caractère préliminaire de ces résultats, ce type de test semble pouvoir s'utiliser facilement pour comparer la multiplication de $H$. schachtii sur des variétés de crucifères. Il est susceptible d'être amélioré afin de le rendre plus fiable et plus facile à réaliser. Ainsi l'utilisation de larves $\mathrm{L} 2$ comme inoculum réduirait le délai d'observation puisque la sortie massive des larves ne se produit que 3 semaines après l'hydratation des kystes (fig. 1).

Il est intéressant de disposer de crucifères s'opposant à la multiplication de $H$. schachtii car elles peuvent jouer un rôle véritable de plante piège en stimulant l'éclosion, donc en favorisant la pénétration, puis en empêchant la formation de femelles. Pour que de telles variétés soient utiles aux betteraviers, il nous semble indispensable de définir les aptitudes de chacune à réduire, dans les conditions agronomiques d'emploi et mieux qu'une jachère, les populations du ravageur visé. Pour ce faire, il est indispensable que le cultivar choisi assure bien une stimulation notable de l'éclosion et que les expérimentations de terrain prennent en rompte l'évolution des densités de populations.

L'utilisation de telles variétés devra tenir compte aussi de l'effet de la date de semis, de la profondeur de l'enracinement et de l'action sur l'ensemble des ravageurs dont certains pourraient se développer sur la culture ; elle ne pourra donc s'envisager que dans un système de lutte intégrant les autres possibilités d'intervention. En aucun cas, on ne doit se contenter de comparer le rendement d'une culture de betterave après une culture d'engrais vert s'opposant à la multiplication d'H. schachtii avec celui obtenu après jachère, puisqu'il faut pouvoir dissocier l'effet engrais vert et l'effet sur les nématodes.

Reçu le 28 mai 1984. Accepté le 14 janvier 1985.

\section{RÉFÉRENCES BIBLIOGRAPHIQUES}

Baukloch H., 1976. Untersuchung zur Wirtspflanzeneignung der Kruziferen gegenüber dem Rübennematoden, Heterodera schachtii (Schmidt), unter besonderer Berücksichtigung der Resistenzzüchtung. Dissert. Univ. Göttingen, $61 \mathrm{p}$.

Caubel G., 1982. Les nématodes en culture betteravière. Générale Sucrière Culture, 41, 10-15.

Heijbroek W., 1982. The influence of resistant cruciferous green manure crops on beet cyst nematodes. Meded. Inst. ration. suikerprod., 8, 20 p.

Kort J., 1960. A technique for the extraction of Heterodera cysts from wet soil and for the estimation of their egg and larval content. Multilith P.D. Wageningen.

Lubberts J. H., Toxopeus H., 1982. Het ontwikkelen van bietecystenaaltjesresistente bladrammenas en gele mosterd. Zaadbelangen, 36, 66-69.

Müller J., 1978. L'élevage monoxénique de Heterodera schachtii sur crucifères. Application à la sélection de plantes résistantes. Rev. Nématol., 1, 47-52.
Müller J., Von Kries A., 1981. Zum Stand der Resistenzzüchtung gegen Heterodera schachtii beim Ölrettich. Nachrichtenbl. Deutsch. Pflanzenschutzd. (Braunschweig), 33, 122.

Rivoal R., Person F., Caubel G., Scotto la Massese C., 1978. Méthodes d'évaluation de la résistance des céréales au développement des nématodes Ditylenchus dipsaci, Heterodera avenae, Pratylenchus spp. Ann. Amélior. Plantes, 28, 31-54.

Steele A. E., Toxopeus H., Heijbroek W., 1982. A comparison of the hatching of juveniles from cysts of Heterodera schachtii and H. trifolii. J. Nematol., 14, 588-592.

Steudel W., Müller J., 1983. Untersuchugen und Modellrechnungen zum Einfluss pflanzenverträglicher Nematizide und nematodenresistenter Zwischenfrüchte auf die Abundanzdynamik des Zuckerrübennematoden (Heterodera schachtii) in Zuckerrübenfruchtfolgen. Zuckerind., 108, 365-369. 